\title{
Evaluation of ivermectin mass drug administration for malaria transmission control across different West African environments
}

\author{
Haoues Alout ${ }^{1 *}$, Benjamin J Krajacich', Jacob I Meyers ${ }^{1,2}$, Nathan D Grubaugh', Doug E Brackney ${ }^{1}$, \\ Kevin C Kobylinski, ${ }^{3,4}$, Joseph W Diclaro II ${ }^{5}$, Fatorma K Bolay ${ }^{6}$, Lawrence S Fakoli ${ }^{6}$, Abdoulaye Diabaté \\ Roch K Dabiré ${ }^{7}$, Roland W Bougma ${ }^{8}$ and Brian D Foy ${ }^{1}$
}

\begin{abstract}
Background: Mass drug administration (MDA) of ivermectin to humans for control and elimination of filarial parasites can kill biting malaria vectors and lead to Plasmodium transmission reduction. This study examines the degree and duration of mosquitocidal effects resulting from single MDAs conducted in three different West African countries, and the subsequent reductions in parity and Plasmodium sporozoite rates.

Methods: Indoor-resting, blood-fed and outdoor host-seeking Anopheles spp. were captured on days surrounding MDAs from 2008-2013 in Senegalese, Liberian and Burkinabé villages. Mortality was assessed on a portion of the indoor collection, and parity status was determined on host-seeking mosquitoes. The effect of MDA was then analysed against the time relative to the MDA, the distributed drugs and environmental variables.

Results: Anopheles gambiae survivorship was reduced by 33.9\% for one week following MDA and parity rates were significantly reduced for more than two weeks after the MDAs. Sporozoite rates were significantly reduced by $>77 \%$ for two weeks following the MDAs in treatment villages despite occurring in the middle of intense transmission seasons. These observed effects were consistent across three different West African transmission dynamics.

Conclusions: These data provide a comprehensive and crucial evidence base for the significant reduction in malaria transmission following single ivermectin MDAs across diverse field sites. Despite the limited duration of transmission reduction, these results support the hypothesis that repeated MDAs with optimal timing could help sustainably control malaria as well as filarial transmission.
\end{abstract}

Keywords: Mass drug administration, Ivermectin, Transmission, West Africa, Malaria control, Plasmodium falciparum, Anopheles gambiae, Survival, Parity, Environment

\section{Background}

Despite substantial efforts dedicated to control and eliminate malaria from certain regions, it is still a major public health issue. In 2012 nearly 207 million cases occurred with approximately 627,000 deaths, $77 \%$ of which were children younger than five years of age [1]. Current recommendations to combat malaria include artemisinin-based combination therapy (ACT) and long-lasting insecticidal

\footnotetext{
* Correspondence: haoues.alout@colostate.edu

'Arthropod-borne and Infectious Diseases Laboratory, Department of Microbiology, Immunology and Pathology, Colorado State University, Fort Collins, CO, USA

Full list of author information is available at the end of the article
}

nets (LLIN), supported by indoor-residual spraying of insecticide (IRS) and intermittent preventive treatment during pregnancy. Deployment of these strategies has fostered important reductions of malaria-associated morbidity and mortality in settings with moderate-to-high transmission levels in sub-Saharan Africa [2]. However, widespread insecticide resistance in vectors [3], increasing malaria cases in some African countries [1,4], and concern over spreading artemisinin resistance [5], underlie the fragility of malaria prevention and control. To ensure the success of malaria elimination, the Roll Back Malaria Partnership and Malaria Eradication Research Agenda (malERA) consultative vector control group emphasized the need to search

\section{Ciomed Central}

(c) 2014 Alout et al.; licensee BioMed Central Ltd. This is an Open Access article distributed under the terms of the Creative Commons Attribution License (http://creativecommons.org/licenses/by/4.0), which permits unrestricted use, distribution, and reproduction in any medium, provided the original work is properly credited. The Creative Commons Public Domain Dedication waiver (http://creativecommons.org/publicdomain/zero/1.0/) applies to the data made available in this article, unless otherwise stated. 
for innovative strategies [6]. These strategies should aim at developing new insecticides with novel modes of action, developing effective control methods for outdoor-feeding and resting vectors, and sustaining and integrating novel interventions in order to significantly decrease and even interrupt disease transmission in endemic areas.

Ivermectin is an endectocide that has been extensively used alone for decades for the control of onchocerciasis, or in combination with albendazole for the elimination of lymphatic filariasis. Currently, more than 300 million individuals living in areas endemic for filarial infections are treated each year in mass drug administration (MDA) campaigns [7]. The drug has an excellent safety profile in humans and can be lethal to mosquitoes when they feed on treated humans. Ivermectin MDA addresses specific malERA recommendations including: a) a different mode of action from current insecticides; b) it targets all biting vectors, regardless of their ecology and feeding behaviour; and, c) it may be integrated into existing strategies to simultaneously control malaria, filariasis and other neglected tropical diseases [8]. The mosquitocidal effect of ivermectin MDA has been demonstrated on several Anopheles species from field trials $[9,10]$. However, subsequent effects on vector population age-structure has only been modelled [11], and effects on vector infection rates with Plasmodium have only been measured for a limited duration in one setting [12]. Repeated MDAs with ivermectin have been proposed as a complementary Plasmodium transmission control tool [10] but several knowledge gaps need to be filled in order to fully evaluate this strategy. Here, the effects of single ivermectin MDAs were comprehensively analysed across different years and in three West African countries with distinct malaria transmission dynamics: Senegal, Liberia and Burkina Faso (Figure 1, Table 1). The degree and duration of effects on mosquito survival, parity rate and the proportion of sporozoiteinfected vectors before and after single ivermectin MDAs in treatment villages, and in pair-matched, untreated villages, were assessed, taking into account several biotic (species, exophily) and abiotic (environmental) factors.

\section{Methods}

\section{Ethical statement}

The study has been reviewed and approved by human subjects' research reviews in each country (Senegal, Etude des vecteurs $d u$ paludisme en zone onchocerquienne au Senegal; Liberia, EC/LIBR/012/033; Burkina Faso, 282013/CE-CM) in compliance with the Helsinki Declaration. Informed consent was obtained from all adult and paid mosquito collectors. Human subject research protocols $11-3121 \mathrm{H}$ and $11-2874 \mathrm{H}$ were also approved by the Colorado State University Institutional Review Board.

\section{Study sites}

Mosquito sampling was conducted during five collection periods in three West African countries: Senegal, Liberia and Burkina Faso (Table 1). Senegal: sampling occurred in 2008, 2009 and 2012 in the southeastern villages of Boundacoundi, Damboucoye, Nathia, Ibel, and Ndebou. Bed net coverage ranged from $78.2 \%$ in 2009 to $82.0 \%$ in 2012. Select villages in this region are treated by MDA with $150 \mu \mathrm{g} / \mathrm{kg}$ of ivermectin alone (Mectizan ${ }^{\oplus}$, Merck \& Co Inc) for onchocerciasis control. Liberia: sampling occurred in 2013 in the village of Ngaisaikoryah (Foya District, Lofa County) where the bed net coverage was $38.3 \%$. MDA was designed to control lymphatic filariasis (LF), onchocerciasis and soil-transmitted helminths with a combination of ivermectin $(150 \mu \mathrm{g} / \mathrm{kg})+$ albendazole (400 mg) (Albenza ${ }^{\circ}$, GlaxoSmithKine). Burkina Faso: sampling occurred in 2013 in the villages of Bougouriba (Haut-Bassins region, control) and Diarkadougou East (Sud-Ouest region, treated) where the average bed net coverage was $42.0 \%$. MDA was designed to control LF with a combination of ivermectin $(150 \mu \mathrm{g} / \mathrm{kg})+$ albendazole (400 mg). MDA coverage rates in treatment villages were $82.1-84.0 \%$ in Senegal, $76.2 \%$ in Liberia and $83.0 \%$ in Burkina Faso, and were recorded either directly by investigators or provided by health authorities.

\section{Mosquito collections and processing}

Mosquito collections were performed by aspiration of indoor-resting, blood-fed mosquitoes on select mornings, as described by Sylla et al. [10]. Collected bloodfed females were kept in field insectaries, which were designated rooms of houses that had screened and slatted windows so that they naturally fluctuated with the ambient temperature and humidity. Temperature and relative humidity were monitored daily every 15 minutes in insectaries using a $\mathrm{HOBO}^{\circ}$ device (Onset Computer Corporation) to determine the daily fluctuation of temperature and hygrometry, respectively. A subset of approximately 50 fully engorged Anopheles gambiae s.l. was transferred into cardboard cups covered with a mesh with access ad libitum to a sugar solution for survival analysis. Dead mosquitoes were counted daily, removed from cups over five consecutive days and identified morphologically to species. The head + thoracies of mosquitoes that survived after five days were stored individually in $1.5-\mathrm{ml}$ tubes containing desiccant. Remaining collected mosquitoes were dissected on the same day of capture and head + thoracies were stored individually in 1.5-ml tubes with desiccant.

Additionally, outdoor host-seeking mosquitoes were captured over the night prior to morning house aspirations by either human-landing catch or tent traps, as previously described in Krajacich et al. [14]. These were identified morphologically to species and dissected for head + thoracies 

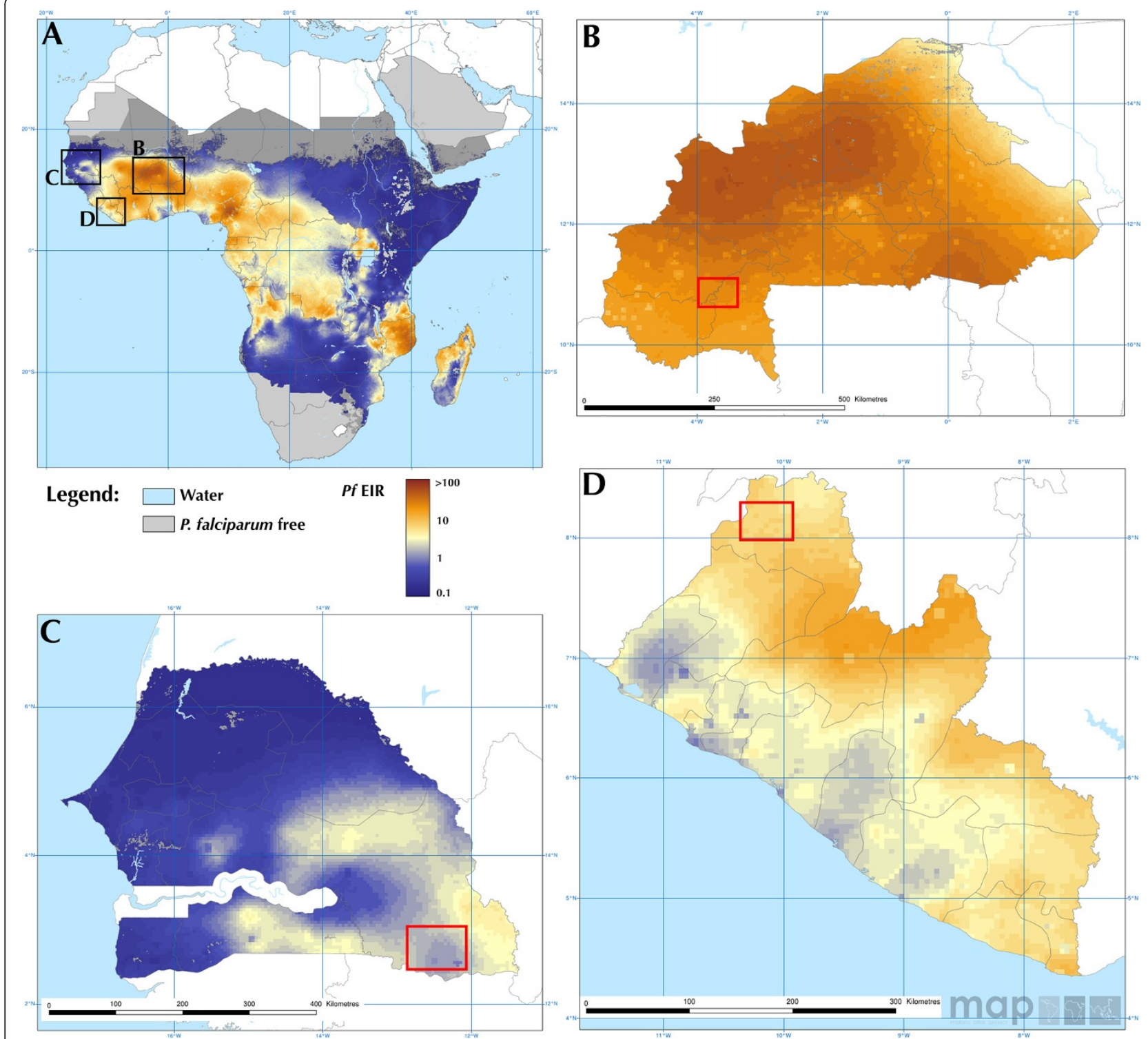

Figure 1 Study sites outlined on maps showing the spatial distribution of the Plasmodium falciparum entomological inoculation rate (PfEIR) in Africa (panel A), Burkina Faso (B), Senegal (C) and Liberia (D) in 2010. Red squares represent the location of the sampled villages. Data are available at Malaria Atlas Project [13].

on the same day as capture as described above. A subset of these mosquitoes was also used for parity analysis.

\section{Parity determination}

Age grading of An. gambiae s.l. was determined by parity analysis in samples from Senegal 2012 and from Burkina
Faso 2013. A random batch of approximately 20 females from each village and each sampling day was dissected for their ovaries. Ovaries were dissected in water under a light microscope and allowed to dry. Parity rate was determined by observing the presence of coiled or uncoiled ovarian tracheoles [15].

Table 1 Characteristics of study sites

\begin{tabular}{|c|c|c|c|c|c|}
\hline Country & Phytogeographic zone & Rainy season & Malaria endemicity & MDA treatment & Diseases targeted \\
\hline Burkina Faso & Sudanian & June-October & Hyperendemic & $I V M+A L B$ & LF \\
\hline Liberia & Tropical rainforest & Year round & Holo-endemic & $I V M+A L B$ & LF, NTD, onchocerciasis, \\
\hline Senegal & Sudano-Guinean & May-October & Hyperendemic & IVM & Onchocerciasis \\
\hline
\end{tabular}




\section{Mosquito species determination and Plasmodium spp. detection}

DNA was extracted with the Qiagen DNeasy kit and was used to identify the species in the An. gambiae complex [16]. DNA from individual head + thoraxes were tested by Taqman polymerase chain reaction for Plasmodium spp. sporozoite detection [17], which used laboratoryconfirmed Plasmodium falciparum sporozoite-infected An. gambiae s.s., as positive controls.

\section{Statistical analysis \\ Mosquito survivorship}

Anopheles gambiae survivorship was analysed using a generalized linear mixed-effect model with a binomial error structure to compare the effect of MDA between West African countries. The effect of eight variables on mosquito survivorship was analysed. These included the categorical variables field sites (Senegal 2008, 2009-mid season and 2009-end season, Liberia 2013 and Burkina Faso 2013), the village (treated or control), the treatment type (ivm or $i v m+a l b)$, the species, the collection (indoor or outdoor), the numerical variables temperature and hygrometry fluctuations (i.e., the difference between the maximum and the minimum values on each sampling day), and time relative to MDA date. The binary response variable was mosquito survivorship, counted as either dead or alive for each individual on the third day post-capture, when the maximal reduction in mosquito survivorship is observed [18]. Two types of analyses were performed: one to compare the difference in survival rate over time (in weeks) between treated and control villages and another to characterize the difference in survival rate over time (in weeks) between the treatment types. The first analysis was performed on all data obtained from Senegal and Burkina Faso only because there were no untreated villages during the sampling period in Liberia. This analysis included data from 3,140 An. gambiae s. $l$. and tested the influence of the site, village, species, collection, temperature and hygrometry fluctuations and time. The second analysis included data from 1,370 An. gambiae s.l., collected from treated villages only (including Ngaisakoryah, Liberia) and collected after the date of drug distribution only, and tested the influence of site, treatment type, species, collection, temperature and hygrometry fluctuation and time. The site variable was used as a random variable to account for the nested data structure, i.e., the correlation between individuals from the same field site. For each analysis, the random structure was selected and compared with a generalized linear model with no random effect based on the lowest Akaike's information criterion (AIC). Statistical analyses were performed with Statistical Analysis Software (SAS Institute Inc., Cary, NC, USA) using the GLIMMIX procedure. This procedure performs a type III hypothesis for the fixed effect variables and computes the $F$-statistic based on Satterthwaite's approximation. The maximal models included all the variables with interactions and were subsequently simplified following a step-by-step AIC-based procedure. Output of the minimal model was used to produce the local regression (LOESS procedure) with the computed 95\% confidence intervals. The mean survival rates by week were computed and compared between villages (control and treated) taking into account multiple testing using the Bonferroni procedure.

\section{Parity rate}

Parity data were analysed weekly and significant differences in parity rate over time were assessed separately for the control $(\mathrm{N}=327)$ and the treated $(\mathrm{N}=380)$ villages, and also between villages for each week, separately (pre-MDA: $\mathrm{N}=222$, week $1: \mathrm{N}=130$, week $2: \mathrm{N}=112$, week 3: $\mathrm{N}=243$ ) using the $\chi^{2}$-test. The parity data were analysed also with a generalized linear model with a binomial error structure (GLM procedure in SAS) to produce the local regression (LOESS procedure) with the computed $95 \%$ confidence intervals.

\section{Sporozoite rate}

Variations in sporozoite rates over time were analysed only from data obtained in Senegal 2012, Liberia 2013 and Burkina Faso 2013 reported here. Analysis was performed using a generalized linear model with a binomial error structure (GLM procedure in SAS), due to the low number of field sites included. The effect of seven variables on mosquito sporozoite rate was analysed: site, village, species, collection as categorical variables and temperature and hygrometry fluctuations and time relative to MDA (in weeks) as numerical variables. The binary response variable was the status of Plasmodium infection in thoraces (i.e., infected or uninfected). The model was subsequently simplified following a stepwise AIC-based procedure. Significance of the variable retained in the minimal model was determined using the type III test and normality of residuals was checked. Output of the minimal model was used to produce the local regression (LOESS procedure) with the computed $95 \%$ confidence intervals.

\section{Results}

\section{Effect of MDA on Anopheles gambiae survival rate}

The three-day survival rate of An. gambiae s.l. collected in each field site is summarized in Table 2 by sampling week. The three-day survival rate was first analysed in treated villages and compared with that from controls, so only data from Burkina Faso and Senegal (2008 and 2009) were used because a control village in Liberia was not sampled. The influence of field sites appeared to be weak as the variance of the random variable site 
Table 2 Three-day survival rate of wild caught Anopheles gambiae s.l. following mass drug administration of ivermectin in the treated village compared to the control village

\begin{tabular}{|c|c|c|c|c|c|c|c|c|}
\hline \multirow[t]{2}{*}{ Study site } & \multirow[t]{2}{*}{ Year } & \multirow[t]{2}{*}{ Village } & \multicolumn{6}{|c|}{ Time relative to MDA (week) } \\
\hline & & & Week -3 & Week -2 & Week -1 & Week 1 & Week 2 & Week 3 \\
\hline \multirow[t]{3}{*}{ Burkina Faso } & 2013 (Aug.-Sept.) & Control & $0.96 \pm 0.02(89)$ & $0.70 \pm 0.05(70)$ & NA & $0.71 \pm 0.03(266)$ & $0.651 \pm 0.04(129)$ & $0.89 \pm 0.05(45)$ \\
\hline & & Treated & $0.85 \pm 0.04(75)$ & $0.75 \pm 0.04(122)$ & NA & $0.50 \pm 0.03$ & $0.709 \pm 0.03(196)$ & $0.81 \pm 0.06(41)$ \\
\hline & & $p$-value & 0.0477 & 0.6029 & - & $1.54 E-07$ & 0.3268 & 0.4325 \\
\hline \multirow[t]{3}{*}{ Liberia } & 2013 (June) & Control & NA & NA & NA & NA & NA & NA \\
\hline & & Treated & NA & $0.98 \pm 0.01(104)$ & $0.91 \pm 0.04(66)$ & $0.42 \pm 0.04(147)$ & $0.86 \pm 0.05(50)$ & NA \\
\hline & & $p$-value & - & - & - & - & - & - \\
\hline \multirow[t]{9}{*}{ Senegal } & 2009 (Oct.) & Control & NA & NA & $0.83 \pm 0.06(40)$ & $0.81 \pm 0.05(62)$ & $0.792 \pm 0.08(24)$ & NA \\
\hline & & Treated & NA & NA & $0.79 \pm 0.02(338)$ & $0.48 \pm 0.04(168)$ & $0.75 \pm 0.06$ & NA \\
\hline & & $p$-value & - & - & 0.7879 & $1.5 E-05$ & 0.9218 & - \\
\hline & 2009 (July-Aug.) & Control & NA & $0.77 \pm 0.08$ & $0.61 \pm 0.09$ & $0.72 \pm 0.06(60)$ & $0.833 \pm 0.07(30)$ & NA \\
\hline & & Treated & NA & $0.90 \pm 0.04$ (59) & $0.82 \pm 0.03(154)$ & $0.57 \pm 0.04(150)$ & $0.819 \pm 0.03(160)$ & NA \\
\hline & & $p$-value & - & 0.2162 & 0.015 & 0.077 & 1 & - \\
\hline & 2008 (Aug.) & Control & NA & NA & $0.76 \pm 0.07(37)$ & $1.0 \pm 0(26)$ & $0.842 \pm 0.08(19)$ & NA \\
\hline & & Treated & NA & NA & $0.70 \pm 0.05$ & $0.40 \pm 0.06(70)$ & $0.636 \pm 0.15$ & NA \\
\hline & & $p$-value & - & - & 0.6427 & $4.78 E-07$ & 0.4031 & - \\
\hline
\end{tabular}

Data presented are mean survival rate \pm s.e. with sample size in brackets. Significance of differences ( $p$-value) between the control and the treated villages were assessed using $x^{2}$ test, $p$-value below 0.05 are indicated in bold.

is $0.05571 \pm 0.05186$ (estimate \pm standard error), but still distinct from zero. Mosquito survival rate was significantly influenced by the variables: village $\left(F_{1,3434}=5.48, p=\right.$ $0.0193)$, time $\left(F_{2,3434}=21.22, p<0.001\right)$, hygrometry fluctuation $\left(F_{1,3434}=9.66, p=0.0019\right.$; Additional file 1: Figure $\mathrm{S} 1)$, and the interaction of village with time $\left(F_{2,3434}=\right.$ 13.62, $p<0.001)$. Neither the species nor the exophily were retained in the minimal model. The computed three-day survival rate of An. gambiae s.l. over time in the treated and the control villages from all field sites shows a reduction during the first week after MDA from $82.3 \% \pm 2.0$ in the control villages to $54.4 \% \pm 3.2$ in the treated villages (a 33.9\% reduction over the three days). Hygrometry fluctuation negatively influenced the mosquito survival rate (Spearman correlation coefficient; $-0.5253, p<0.0001$; Additional file 1: Figure S1).

\section{Comparison of MDA treatments on the survival rate of Anopheles gambiae}

This analysis focused only on treated villages to characterize the duration of the reduction in survival rate post-treatment, as well as the potential effect of the different drug regimens (either ivermectin + albendazole or ivermectin alone), thus it included data from all three ecological settings starting from the date of MDA. As expected, the influence of time is significant because survival rate increased over time to the pretreatment level $\left(F_{1,1361}=73.72, p<0.001\right)$. Interestingly, the effect of time was different between treatment types (treat. type by time interaction: $F_{1,1361}=5.36, p=0.0208$ ), suggesting that the ivermectin + albendazole treatment may have resulted in an apparent longer-lasting mosquitocidal effects as compared to treatment with ivermectin alone. However, the degree of reduction in mosquito survival rate did not differ between treatment types: from an average of $88.2 \% \pm 2.6$ before MDA to $53.44 \% \pm 3.4$ for ivermectin alone, compared to $59.69 \% \pm 2.4$ for ivermectin + albendazole, the week following MDA $(p=0.809)$. The hygrometry fluctuation did not significantly influence the overall survival rate $\left(F_{1,1361}=0.22, p=0.6427\right)$, but the analysis revealed a significant interaction of hygrometry variation with treatment type (Hygro. by Treat. interaction: $\left.F_{1,1361}=5.85, p=0.0157\right)$.

\section{Parity rate}

Figure 2 represents the variation in parity rate by week and shows no significant variation over time in the control village $\left(\chi_{\mathrm{df}=3}^{2}=3.96, p=0.265, \mathrm{~N}=327\right)$, ranging between 80 and $89.72 \%$ (mean of $85.4 \% \pm 2.3$ ). In contrast, the proportion of parous female $A n$. gambiae s.l. varied significantly over time in the treated village $\left(\chi_{\mathrm{df}=3}^{2}=14.36\right.$, $p=0.0024, \mathrm{~N}=380$ ). While $80.7 \% \pm 3.6$ of host-seeking females were parous before MDA in the treatment village and showed no significant difference with the control village $\left(\chi_{\mathrm{df}=1}^{2}=0.033, p=0.856, \mathrm{~N}=222\right)$, this proportion significantly decreased to $60.0 \% \pm 5.6\left(\chi_{\mathrm{df}=1}^{2}=4.98, p=0.0255\right.$, $\mathrm{N}=130$ ) over the first week post-MDA and to $58.0 \% \pm 7.0$ $\left(\mathrm{N}=50, \mathrm{X}_{\mathrm{df}=1}^{2}=5.78, p=0.0161, \mathrm{~N}=112\right)$ over the second week post-MDA. By the third week post-MDA, the parity 


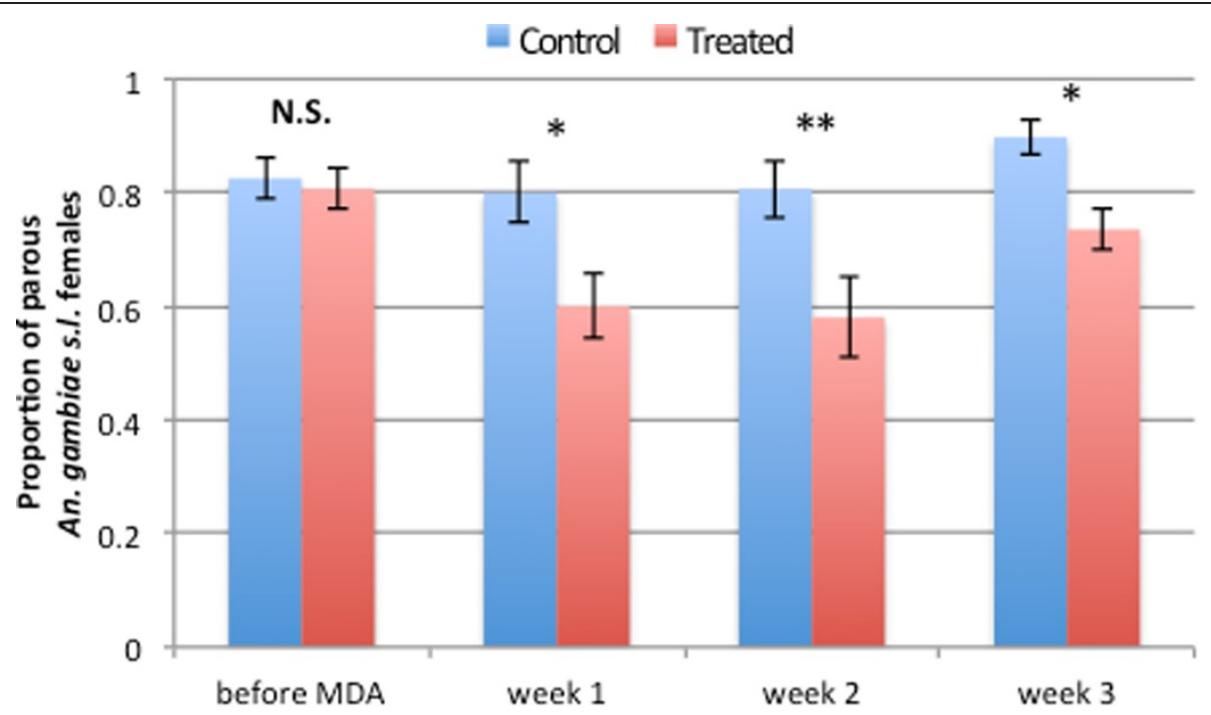

Figure 2 Mosquito parity rate over time in treated and control villages. Significant differences in parity rates between the control (blue bars) and the treated villages (red bars) are denoted by stars above the column pairs and derived using a Chi-squared test (N.S. $=$ not significant). Error bars are the standard error of the mean. Sample sizes were 103 and 119 pre-MDA, 55 and 75 on week 1, 62 and 50 on week 2 and 107 and 136 on week 3 in the control and the treated villages, respectively.

rate in the treated villages increased to $73.5 \% \pm 3.8$ but remained significantly lower than in the control villages $\left(x_{\mathrm{df}=1}^{2}=9.05, p=0.0026, \mathrm{~N}=243\right)$.

\section{Sporozoite rate}

A proportion of all vectors were processed on the day of capture from Senegal 2012, Liberia 2013 and Burkina Faso 2013 (Additional file 2: Table S1) and analysed for sporozoite infections over time. Overall, the variable time did not significantly influence the sporozoite rate $\left(x_{\mathrm{df}=5}^{2}=8.198, p=0.1456\right)$. However, the variation across time is significantly different between villages (time: village interaction: $\mathrm{X}_{\mathrm{df}=5}=25.89, p<0.001$ ), indicating a difference between control and treated villages. The mean sporozoite rate in the control villages did not significantly change over time, varying from $2.71 \% \pm 0.8$ to $4.84 \% \pm 0.9(p=1)$. In the treated villages, ivermectin MDA reduced the sporozoite rate from $5.31 \% \pm 1.2$ pre-MDA to $2.03 \% \pm 0.8$ the first week $(p=$ 0.0074 ) and to $1.19 \% \pm 0.7$ the second week after MDA $(p=0.0018)$. In addition, the significant site:time interaction $\left(\chi_{\mathrm{df}=5}^{2}=29.25, p<0.001\right)$ indicated that the variation of sporozoite rate across time is significantly different between malaria ecologies. However, the minimal model did not retain the time:site:village triple interaction, indicating that the difference between control and treated villages over time is consistent among field sites. Interestingly, sporozoite rate was also significantly influenced by daily temperature fluctuation $\left(\mathrm{x}^{2}\right.$ $\mathrm{df}=1=7.334, p=0.0068$; Additional file 3: Figure S2).

\section{Combined mosquito survival, parity and sporozoite rate} over time

Figure 3 shows the dynamic of survival, parity and sporozoite rates across time (individual sampling days) relative to the MDA date, obtained by local regression of all relevant data. For most of the pre-MDA sampling dates, the control and treated villages do not significantly differ in survival rate (Figure 3A) and parity rate (Figure 3B) as evidenced by overlapping $95 \%$ confidence intervals. Over this same time interval, the mosquito sporozoite rate (Figure $3 \mathrm{C}$ ) from treated villages was significantly greater than from pair-matched control villages, and fluctuated more significantly between sites and villages, as evidenced by more broad $95 \%$ confidence intervals, likely due to the variable nature of human-to-mosquito transmission. Immediately following MDA in the treated villages, all three measures were significantly reduced in the treated villages compared to control villages. Survival rate in treated villages was the first measure to recover to pre-MDA and control village values, at day 7 post-MDA. The parity rate never recovered to pre-MDA and control village values by the end of the sampling, but it did hit a slower increasing plateau after 15 days post-MDA (dotted line on the right of all graphs). On this same date postMDA, the sporozoite rate in treated villages recovered to the same rate as pre-MDA and control villages.

\section{Discussion}

In an attempt to comprehensively evaluate the effects of single ivermectin MDA, alone or in combination 

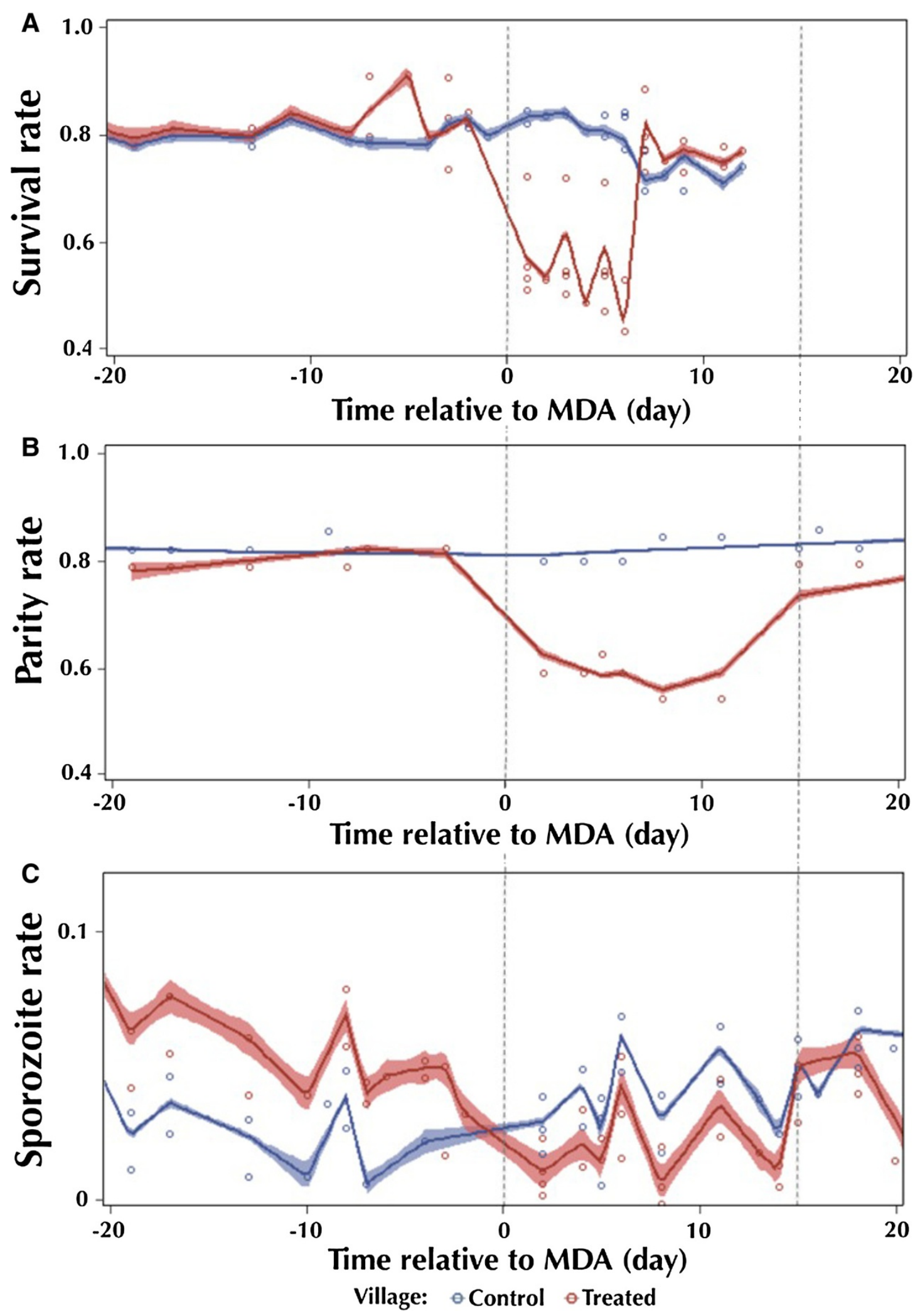

Figure 3 Dynamic of survival (A), parity (B), and sporozoite (C) rates across time relative to mass drug administration. Daily data points represented as circles from control (blue) and treated (red) villages, with the computed $95 \%$ confidence intervals using the local regression method (LOESS) in SAS software. 
with albendazole, on malaria transmission, their effect on natural populations of mosquito vectors was characterized in three West African countries with different malaria ecologies. The primary first order effect of ivermectin MDA was on mosquito survival rate, which was consistent across all sites. While lasting a relatively short time (one week), the observed lethal effect of ivermectin on the vector population biting the villagers is strong with respect to vectorial capacity. The calculated reduction in daily mosquito survival rate $(p)$ of $11 \%$ would lead to a $78 \%$ reduction in vectorial capacity for the week following the MDA [19]. Nonetheless, recent modelling of the impact of ivermectin on malaria transmission demonstrated that the duration of the mosquitocidal effect has a greater impact than its magnitude [20]. The two different drug regimens did not differently affect the degree of mosquito survival rate change, suggesting no additional effect from albendazole, which is consistent with published laboratory results [18]. While differences in field sites did not appear to strongly influence the model, this analysis seems to indicate that the ivermectin + albendazole regimen resulted in a longer mosquitocidal effect compared to the ivermectin alone regimen (significant treat. type by time interaction). Awadzi et al. [21] found no significant differences in ivermectin plasma pharmacokinetics when administered with albendazole, potentially ruling out drug-drug interactions in humans, however drug-drug interactions in the mosquito might be a potential factor. From this study, it is not clear what is influencing this observation and further experiments are needed to characterize the role of other biotic or abiotic factors affecting vector population susceptibility.

Modelling predicted that the mortality effects from a single MDA would temporarily shift the population structure of vector mosquitoes around treated villages [11]. In concordance with this model, the proportion of parous, outdoor host-seeking mosquitoes in the populations studied was reduced by $25 \%$. This reduction is equivalent to parity rate changes observed in An. gambiae populations after implementation of IRS with dichlorodiphenyltrichloroethane (DDT) [22] and LLIN distribution [4]. The observed reduction in parity rate may, in part, be due to an increased susceptibility to ivermectin in older mosquitoes, a phenomenon that has been reported with the use of other insecticides, including pyrethroids and DDT [23]. Such an effect would lead to a greater impact on malaria transmission, as mosquito vectors need to survive the extrinsic incubation period to become infectious (approximately ten days). The one-week reduction of survival rates resulted in a significant shift to a younger mosquito population that lasted more than three weeks after the MDA. This suggests: a) that ivermectin concentrations found in human blood after the first week could still selectively kill older and/or infected mosquitoes, and/or, b) that the population returns to its initial age-structure at a relatively slow speed. In line with the first hypothesis, Kobylinski et al. showed that sublethal concentrations of ivermectin reduced the proportion of $P$. falciparum (NF-54 strain)-infected An. gambiae at both oocyst and sporozoite stages, suggesting an increased susceptibility of infected mosquitoes [24].

Consistent across field sites, sporozoite rates were reduced by $77.5 \%$ for 15 days. The observed sporozoite rate reductions is likely explained by the combination of various ivermectin effects against factors influencing vectorial capacity, including: a) mosquito survival rate (with possible selective effects against older and/or P. falciparum infected adults); b) vector density relative to hosts (effects on egg-laying ability [25]); c) time between mosquito blood meals (refeeding frequency [18]); and, d) potential effect on vector competence (i.e., ability of vectors to support parasite development). The change in sporozoite rate over time reflects the change in parity rate over time, suggesting that the shift of the age-structure may be the main cause of the reduction of sporozoite prevalence in mosquito vectors. While laboratory studies have suggested an anti-sporogonic effect (inhibition of parasite development in the vector) [24], ongoing semi-field and field studies examining the effect of ivermectin on wild-type mosquitoes and parasites will need to sort out these discrepancies.

The limitations of this study include some unavoidable inconsistencies in sampling and processing regimens across each year and field site; for example, a control village was not concomitantly sampled in Liberia. Even so, a single ivermectin MDA has a clear and dramatic impact on the proportion of infectious mosquitoes for up to 15 days. As previously proposed, repeated ivermectin MDAs would be necessary to have a sustained effect on Plasmodium transmission $[8,11]$. Repeated ivermectin MDAs have been shown to be safe, even at higher doses than currently administered [26]. Such regimens also have the potential to integrate with control of neglected tropical diseases (NTD) such as onchocerciasis, LF, soil-transmitted helminths (strongyloidiasis, ascariasis, trichuriasis, and hookworm), scabies, and lice [27]. Questions remain about how frequent, repeated, ivermectin MDAs would need to occur with respect to when in the transmission season they would be administered. The maximal effect would probably occur if MDAs were concentrated during dry-to-wet season transitions, when the numbers of vectors are low but increasing, and thus most susceptible to population level effects. This may also have the most significant effect on malaria incidence, as these transition seasons are also concomitant with peak seasonal risk of clinical malaria in children living in hyperendemic areas, likely due to acquisition of new Plasmodium clones 
when mosquito biting increases [28]. While repeated ivermectin MDAs would reduce Plasmodium transmission, this effect alone would not reduce or eliminate the human reservoir of Plasmodium parasites. Integration with other control measures is critical. Modelling has shown that combining repeated ivermectin MDAs with anti-malarial drugs (e.g., ACT) would sustain transmission interruption and achieve malaria elimination [20]. Addition of albendazole with ivermectin during MDAs would maximize impact against NTDs and help prevent development of helminth resistance to either drug [29]. Lastly, community-directed treatment platforms for ivermectin delivery have been shown to more cost-effectively and efficiently deliver LLINs [30,31]. A large-scale randomized trial would be required to fully evaluate the implementation of multiple control methods alone or in combination. It would be expected that ivermectin MDAs would synergize with LLIN and IRS control methods to more significantly reduce the proportion of infectious vectors, especially in areas where pyrethroid/DDT resistance in vectors is common. Ivermectin MDAs might particularly help to control malaria transmission from outdoorbiting vectors that are less affected by the latter control methods. Overall, such integrated strategies would control many diseases simultaneously, and should lead to strong and lasting health benefits in the treated communities.

\section{Conclusions}

These data show that single ivermectin MDAs significantly affect mosquito population survival rates, which temporarily changes mosquito population age-structure and results in significantly suppressed sporozoite rates for 15 days after the MDA. The results were collected across multiple years and diverse West African malaria ecologies, and demonstrate a consistent effect despite these differences. These data provide a strong evidence base to develop repeated ivermectin MDA for malaria control and elimination strategies, especially when integrated with complementary malaria and NTD control strategies that all can utilize the very successful community-directed treatment models developed for onchocerciasis and LF control.

\section{Additional files}

Additional file 1: Figure S1. Correlation between Anopheles gambiae s.l. survival rate and hygrometry fluctuation $(\mathrm{dH})$. Shaded area around the regression line is $95 \% \mathrm{Cl}$. Spearman correlation coefficient: $-0.5253, p<$ 0.001 . Dots indicate data from distinct field sites: light green: Senegal 2008, pink: Senegal July-Aug. 2009, dark green: Senegal Oct. 2009, red: Burkina Faso 2013

Additional file 2: Table S1. Sporozoite-infected malaria vectors collected indoor and outdoor. Sample sizes are indicated in brackets. HLC, LTC refer to human-landing catch and light trap catch, respectively.
Additional file 3: Figure S2. Correlation between Anopheles gambiae s.l. sporozoite rate and temperature fluctuation (dT). Shaded area around the regression line is $95 \% \mathrm{Cl}$. Pearson correlation coefficient: $0.38360, p<$ 0.001 . Dots indicate data from distinct field sites: green: Senegal 2012, blue: Liberia 2013, red: Burkina Faso 2013.

\section{Abbreviations}

MDA: Mass drug administration; ACT: Artemisinin-based combination therapy; LLIN: Long-lasting insecticidal nets; IRS: Indoor-residual spraying; malERA: Malaria eradication research agenda; LF: Lymphatic filariasis; IVM: Ivermectin; ALB: Albendazole; AIC: Akaike's information criterion; GLM: General linearized model; DDT: Dichlorodiphenyltrichloroethane; NTD: Neglected tropical diseases; PfEIR: Plasmodium falciparum entomological inoculation rate.

\section{Competing interests}

The authors declare that they have no competing interests.

\section{Authors' contributions}

BDF designed the experiments. HA, BJK, JIM, NDG, DEB, KCK, LSF and BDF performed sampling. $H A, B J K, K C K$, and BDF performed the experiments. $H A$ and BDF analysed the data. BDF, JWD, FKB, LSF, AD, RKD, and RWB contributed reagents/materials tools. HA and BDF wrote the paper. All authors read and approved the final manuscript.

\section{Acknowledgements}

We thank the local people in Senegal, Liberia and Burkina Faso for participating in this study, as well as the local authorities for their support. We are very grateful to the IRSS staff in Burkina Faso, to collaborators in Senegal and to the LIBR staff in Liberia for their technical assistance. We thank Infoscitex Corp for the use of their tent traps in our studies. We thank Jon Seaman, Meg Gray, Timothy Burton, and Wojtek Kuklinski for their assistance in mosquito processing. We are very thankful to Peter U Fischer for discussion and comments. We acknowledge and are grateful for support from National Institute of Allergy and Infectious Diseases grants R21-Al079528 and R01Al094349-01A1, US Armed Forces Health Surveillance Center: Global Emerging Infections Surveillance Network, and Grand Challenges Explorations grant 51995 from the Bill and Melinda Gates Foundation. The funding sources had no role in study design, data collection and analysis, decision to publish, or preparation of the manuscript.

\section{Disclaimer}

The views expressed in this article are those of the authors and do not necessarily reflect the official policy or position of the Department of the Navy, Department of Defense, nor the U.S. Government. Few co-authors are military service members of the U.S. Government.

This work was prepared as part of our official duties. Title 17 U.S.C. §105 provides that 'Copyright protection under this title is not available for any work of the United States Government.' Title 17 U.S.C. §101 defines a U.S. Government work as a work prepared by a military service member or employee of the U.S. Government as part of that person's official duties.

\section{Author details}

${ }^{1}$ Arthropod-borne and Infectious Diseases Laboratory, Department of Microbiology, Immunology and Pathology, Colorado State University, Fort Collins, CO, USA. ${ }^{2}$ Department of Biomedical Sciences, Colorado State University, Fort Collins, CO, USA. ${ }^{3}$ Walter Reed Army Institute of Research, 503 Robert Grant Ave, Silver Spring, MD, USA. ${ }^{4}$ Armed Forces Research Institute of Medical Sciences, 315/6 Rajvithi Road, Bangkok, Thailand. ${ }^{5}$ US Naval Medical Research Unit No 3, Cairo, Egypt. 'Liberian Institute for Biomedical Research, Charlesville, Liberia. ${ }^{7}$ Institut de Recherche en Sciences de la Santé (IRSS), Direction Régionale de l'Ouest (DRO), Bobo Dioulasso, Burkina Faso. ${ }^{8}$ Ministry of Health, Ouagadougou, Burkina Faso.

Received: 30 August 2014 Accepted: 25 October 2014 Published: 3 November 2014

\section{References}

1. WHO: World Malaria Report. Geneva: World Health Organization; 2013. 
2. Pluess B, Tanser FC, Lengeler C, Sharp BL: Indoor residual spraying for preventing malaria. Cochrane Database Syst Rev 2010, 4:CD006657.

3. Ranson H, N'Guessan R, Lines J, Moiroux N, Nkuni Z, Corbel V: Pyrethroid resistance in African anopheline mosquitoes: what are the implications for malaria control? Trends Parasitol 2011, 27:91-98.

4. Trape J-F, Tall A, Diagne N, Ndiath O, Ly AB, Faye J, Dieye-Ba F, Roucher C, Bouganali C, Badiane A, Sarr FD, Mazenot C, Touré-Baldé A, Raoult D, Druilhe P, Mercereau-Puijalon O, Rogier C, Sokhna C: Malaria morbidity and pyrethroid resistance after the introduction of insecticide-treated bednets and artemisinin-based combination therapies: a longitudinal study. Lancet 2011, 11:925-932.

5. Mita T, Tanabe K: Evolution of Plasmodium falciparum drug resistance: implications for the development and containment of artemisinin resistance. Jpn J Infect Dis 2012, 65:465-475.

6. The malERA Consultative Group on Vector Control: A research agenda for malaria eradication: vector control. PLoS Med 2011, 8:e1000401.

7. Mectizan Donation Program: Mectizan Annual Highligths; 2013.

8. Chaccour CJ, Kobylinski KC, Bassat Q, Bousema T, Drakeley C, Alonso P, Foy $\mathrm{BD}$ : Ivermectin to reduce malaria transmission: a research agenda for a promising new tool for elimination. Malar J 2013, 12:153.

9. Bockarie MJ, Hii JL, Alexander N, Bockarie F, Dagoro H, Kazura JW, Alpers MP: Mass treatment with ivermectin for filariasis control in Papua New Guinea: impact on mosquito survival. Med Vet Entomol 1999, 13:120-123.

10. Sylla M, Kobylinski KC, Gray M, Chapman PL, Sarr MD, Rasgon JL, Foy BD: Mass drug administration of ivermectin in south-eastern Senegal reduces the survivorship of wild-caught, blood fed malaria vectors. Malar J 2010, 9:365.

11. Foy BD, Kobylinski KC, da Silva IM, Rasgon JL, Sylla M: Endectocides for malaria control. Trends Parasitol 2011, 27:423-428.

12. Kobylinski KC, Sylla M, Chapman PL, Sarr MD, Foy BD: Ivermectin mass drug administration to humans disrupts malaria parasite transmission in Senegalese villages. Am J Trop Med Hyg 2011, 85:3-5.

13. Gething PW, Patil AP, Smith DL, Guerra C a, Elyazar IRF, Johnston GL, Tatem AJ, Hay SI: A new world malaria map: Plasmodium falciparum endemicity in 2010. Malar J 2011, 10:378

14. Krajacich BJ, Slade JR, Mulligan RF, LaBrecque B, Alout H, Grubaugh ND, Meyers JI, Fakoli LS, Bolay FK, Brackney DE, Burton TA, Seaman JA, Sylla M, Diclaro JW II, Dabiré RK, Foy BD: Sampling host-seeking anthropophilic mosquito vectors in West Africa: comparisons of an active human-baited tent-trap against gold standard methods. Am J Trop Med Hyg. In press.

15. Detinova TS: Age-grouping methods in Diptera of medical importance with special reference to some vectors of malaria. Monogr Ser World Health Organ 1962, 47:13-191.

16. Fanello C, Santolamazza F, della Torre A: Simultaneous identification of species and molecular forms of the Anopheles gambiae complex by PCRRFLP. Med Vet Entomol 2002, 16:461-464.

17. Bass C, Nikou D, Blagborough AM, Vontas J, Sinden RE, Williamson MS, Field LM: PCR-based detection of Plasmodium in Anopheles mosquitoes: a comparison of a new high-throughput assay with existing methods. Malar J 2008, 7:177.

18. Kobylinski KC, Deus KM, Butters MP, Hongyu T, Gray M, da Silva IM, Sylla M Foy BD: The effect of oral anthelmintics on the survivorship and re-feeding frequency of anthropophilic mosquito disease vectors. Acta Trop 2010, 116:119-126.

19. Koella JC: On the use of mathematical models of malaria transmission. Acta Trop 1991, 49:1-25.

20. Slater HC, Walker PGT, Bousema T, Okell LC, Ghani AC: The potential impact of adding ivermectin to a mass treatment intervention to reduce malaria transmission: a modelling study. J Infect Dis 2014:jiu351.

21. Awadzi K, Edwards G, Duke BOL, Opoku NO, Attah SK, Addy ET, Ardrey AE, Quartey BT: The co-administration of ivermectin and albendazole: safety, pharmacokinetics and efficacy against Onchocerca volvulus. Ann Trop Med Parasitol 2003, 97:165-178.

22. Garrett-Jones C: The assessment of insecticidal impact on the malaria mosquito's vectorial capacity, from data on the proportion of parous females. Bull World Health Organ 1964, 31:71-86.

23. Rajatileka S, Burhani J, Ranson H: Mosquito age and susceptibility to insecticides. Trans R Soc Trop Med Hyg 2011, 105:247-253.

24. Kobylinski KC, Foy BD, Richardson JH: Ivermectin inhibits the sporogony of Plasmodium falciparum in Anopheles gambiae. Malar J 2012, 11:381.
25. Fritz ML, Siegert PY, Walker ED, Bayoh MNI, Vulule JRI: Toxicity of bloodmeals from ivermectin-treated cattle to Anopheles gambiae s.l. Ann Trop Med Parasitol 2009, 103:539-547.

26. Kamgno J, Gardon J, Gardon-Wendel N, Demanga-Ngangue, Duke BOL, Boussinesq M: Adverse systemic reactions to treatment of onchocerciasis with ivermectin at normal and high doses given annually or threemonthly. Trans R Soc Trop Med Hyg 2004, 98:496-504.

27. Heukelbach J, Winter B, Wikcke T, Muehlen M, Albrecht S, de Oliveira FA S, Sansigolo Kerr-Pontes LR, Liesenfeld O, Feldmeier H: Selective mass treatment with ivermectin to control intestinal helminthiases and parasitic skin diseases in a severely affected population. Bull World Health Organ 2004, 82:563-571.

28. Mueller I, Schoepflin S, Smith TA, Benton KL, Bretscher MT, Lin E, Kiniboro B, Zimmerman PA, Speed TP, Siba P, Felger I: Force of infection is key to understanding the epidemiology of Plasmodium falciparum malaria in Papua New Guinean children. Proc Natl Acad Sci U S A 2012, 109:10030-10035.

29. Kobylinski K, Alout H, Foy BD, Clements A, Adisakwattana P, Swierczewski B, Richardson J: Rationale for the co-administration of albendazole and ivermectin to humans for malaria parasite transmission control. Am J Trop Med Hyg 2014, 91:655-662.

30. Remme J, The CDI study group: Community-directed interventions for priority health problems in Africa: results of a multicountry study. Bull World Health Organ 2010, 88:509-518.

31. Blackburn BG, Eigege A, Gotau H, Gerlong G, Miri E, Hawley W a, Mathieu E, Richards F: Successful integration of insecticide-treated bed net distribution with mass drug administration in Central Nigeria. Am J Trop Med Hyg 2006, 75:650-655.

doi:10.1186/1475-2875-13-417

Cite this article as: Alout et al.: Evaluation of ivermectin mass drug administration for malaria transmission control across different West African environments. Malaria Journal 2014 13:417.

\section{Submit your next manuscript to BioMed Central and take full advantage of:}

- Convenient online submission

- Thorough peer review

- No space constraints or color figure charges

- Immediate publication on acceptance

- Inclusion in PubMed, CAS, Scopus and Google Scholar

- Research which is freely available for redistribution 